\title{
Ghosts in Discrete Tomography
}

\author{
S. Brunetti - P.Dulio - L.Hajdu - C.Peri
}

\begin{abstract}
Switching components, also named bad configurations, interchanges, and ghosts (according to different scenarios) play a key role in the study of ambiguous configurations, which often appear in Discrete Tomography and in several other areas of research. In this paper we give an upper bound for the minimal size bad configurations associated to a given set $S$ of lattice directions. In the special but interesting case of four directions, we show that the general argument can be considerably improved, and we present an algebraic method which provides such an improvement. Moreover, it turns out that finding bad configurations is in fact equivalent to finding multiples of a suitable polynomial in two variables, having only coefficients from the set $\{-1,0,1\}$. The general problem of describing all polynomials having such multiples seems to be very hard [1]. However, in our particular case, it is hopeful to give some kind of solution. In the context of Digital Image Analysis, it represents an explicit method for the construction of ghosts, and consequently might be
\end{abstract}

\footnotetext{
S.Brunetti

Dipartimento di Ingegneria dell'Informazione e Scienze Matematiche, Università di Siena, Via Roma, 56, 53100 Siena, Italy

E-mail: sara.brunetti@unisi.it

P. Dulio

Dipartimento di Matematica "F. Brioschi", Politecnico di Milano, Piazza Leonardo da Vinci 32, 20133 Milano, Italy

E-mail: paolo.dulio@polimi.it

L. Hajdu

Institute of Mathematics, University of Debrecen, P.O. Box

12, 4010 Debrecen, Hungary

E-mail: hajdul@science.unideb.hu

C. Peri

Università Cattolica S. C., Via Emilia Parmense 84, 29122

Piacenza, Italy

E-mail: carla.peri@unicatt.it
}

of interest in image processing, also in view of efficient algorithms to encode data.

2000 Mathematics Subject Classification. Primary 68U10, 94A08; Secondary 11H71; 05D05; 15A06.

Key words and phrases Bad configuration; Discrete Tomography; generating function; ghost; X-ray.

\section{Introduction}

Discrete tomography (DT) often deals with problems which have application also in different areas, e.g. geometric and computerized tomography, combinatorics, computer vision, image analysis (see for an overview $[2,3]$ and for some recent works [4-6]). Moreover, as a sub-area of Discrete Geometry, DT should also provide both a theoretical and a computational frame-work for digital images ([7]). A basic problem in DT concerns the recovery of an unknown digital image consisting of a small, discrete set of grey values from its X-rays computed along a certain number of directions. Moreover, in the context of DT for finite lattice sets, the term discrete also relates to the nature of X-rays. A discrete parallel X-ray of a finite subset $F$ of $\mathbb{Z}^{n}$, in the direction of a vector $v \in \mathbb{Z}^{n}$, gives the number of points in $F$ lying on each line parallel to $v$. The points in $F$ can model the atoms in a crystal. Electron tomography, and even more modern techniques based on transmission electron microscopy, statistical parameter estimation theory and discrete tomography, effectively allow the discrete parallel X-rays of a crystal to be measured (see for instance [8] and the related bibliography). A main goal of discrete tomography is to use these X-rays to determine the position of the atoms, with a view to applications in the material sciences.

A major point is, of course, in the accuracy of the reconstruction. This is made difficult by the availability 
of limited data since in several applications such as electron tomography or industrial tomography, only few Xrays can be acquired. This prevents the use of the reconstruction algorithms employed in computerized tomography, where the reconstruction problem is a highly undetermined inverse problem. This is due to several noise effects, restrictions and constraints which are introduced when the uniqueness theoretical model, based on the Radon Transform and its inversion formula, is considered in real applications. Even if discrete Filtered Back Projection (FBP) can be exact when the given projection set $S$ tiles the image reconstruction region (and no noise is considered), the general approach provided by FBP points out that any reconstruction process always leads to an approximation of the unknown object. The degree of uncertainty can be quantified in some appearing "sub-pictures" which do not correspond to any really existing structure. A main problem for a correct image understanding is the localization of such sub-pictures.

The same problem arises when FBP is replaced by algebraic reconstruction algorithms, such as ART, SART or SIRT, which usually supply more accurate results in case that only a few projection angles are available (see, for instance $[6,9,10])$. In principle, any algebraic-based reconstruction algorithm, cannot "see" sets having null projections along each one of the employed X-ray directions. Roughly speaking, one has to invert some nonhomogeneous linear system of equations $A x=b$, so that any solution of the homogeneous system $A x=0$ might be superimposed to any reconstruction. Such "pictures" are known as ghost artifacts since X-rays of the ghost image data vanish (or sum up to zero) for a range of pre-selected X-rays angles (see e.g. [11], or [12, Section 16.4]). These can be interpreted as a kind of corruption occurring in the reconstruction process, which is invisible in the available data.

In [13] the generation of ghosts for digital images representing brain cross-sections- is used to illustrate the claim that it is in-practice dangerous to rely on reconstructions from a small number of X-rays, as such a ghost to some extent mimics a malignant tumor, and consequently no reconstruction algorithm could possibly distinguish between the presence and absence of this tumor in the brain.

Of course, with fewer projections, such a phenomenon becomes more significant. Thus, the study of ghost artifacts is of main importance in DT, where the usual approach requires a small number of projections (4 or fewer) so that the choice of the particular set of projection angles can have a large influence on the quality of the reconstructions (see for instance [4,14-18]). The missing information can be only in part overcome by exploiting the knowledge that digital images consist of a low number (2-5) of different materials.

For a prescribed set of directions, the nonexistence of a ghost for a binary image is necessary and sufficient for the X-rays to exactly recognize the image (see $[19,20]$ ). Therefore, ghosts are responsible of ambiguous image reconstructions from the same set of available data and give rise to serious difficulties in image understanding and analysis.

\subsection{Results}

In the context of binary tomography, ghosts first appeared in the reconstruction of binary matrices, under the name of interchanges [21], and later were called switching components [22] or bad configurations. Here it is convenient to represent a digital image as a finite subset of the integer lattice $\mathbb{Z}^{2}$. A simple "cube" construction shows that for any finite set $S$ of lattice directions, it is easy to find two distinct lattice sets that cannot be distinguished by their X-rays in the directions in $S$ (see, for instance, [23]). This leads to the following notion.

Definition 1 An $S$-weakly bad configuration is a pair of lattice sets $(Z, W)$, each consisting of $k$ lattice points, not necessarily distinct (and counted with multiplicity), $z_{1}, \ldots, z_{k} \in Z$ and $w_{1}, \ldots, w_{k} \in W$ such that for each direction $(a, b) \in S$, and for each $z_{r} \in Z$, the line through $z_{r}$ in direction $(a, b)$ contains a point $w_{r} \in W$ (see Figure 1). If all the points in each set $Z$ and $W$ are distinct, then $(Z, W)$ is called $S$-bad configuration (see Figure 2).

We then say that a lattice set $E$ has a (weakly) bad configuration, if an $S$-(weakly) bad configuration $(Z, W)$ exists for some $k \geq 2$, such that $Z \subseteq E, W \subset$ $\mathbb{Z}^{2} \backslash E$.

There is a wide literature concerning (weakly) bad configurations, which highlights their central role in important issues such as ambiguity in the reconstruction problem, or, on the contrary, uniqueness (see, for instance, [24], [25], or [3] and the references given there). For instance, as mentioned above, a set is $S$-unique if and only if it has no $S$-bad configuration. Similarly, a set is $S$-additive (or additive) if and only if it has no $S$-weakly bad configuration (recent examples can be found, for instance, in [26]). Additivity is also of main importance because the reconstruction problem for additive set is polynomial by using (relaxation of integer) linear programming.

A related intriguing problem is to find non-additive sets of uniqueness (see [27]). A complete characterization of 
bad configurations (weakly or not weakly) has been obtained in [25] with a new algebraic approach employed then in several papers (see for instance [27-30]). S-bad configurations, with the extra condition of convexity, are known as $S$-polygons, and reveal to be useful both in geometric tomography and in discrete tomography (see for instance [31,32], and [33] for an algorithmic approach), as well as very interesting also from a purely geometric point of view (see for instance [34-36]).

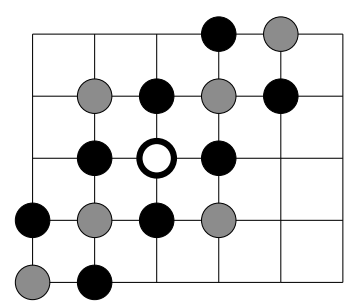

(a)

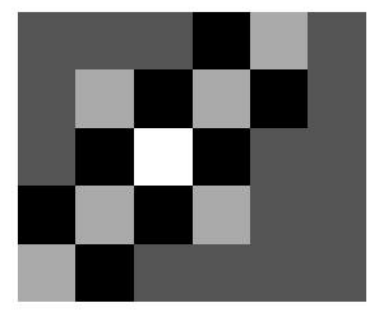

(b)

Fig. 1: (a) A weakly bad configuration associated to $S=\{(1,0),(0,1),(2,1),(1,2)\}$, where $Z$ consists of the grey points and the white point (counted twice), while $W$ is the set of black points. (b) Digital representation of the weakly bad configuration.

In this paper we focus on "minimal" bad configurations. Actually, looking for the smallest possible such configuration is of main interest, since it is related to the problem of finding the largest digital images which are uniquely determined by their X-rays in the X-ray directions. Results concerning the number of points which can be reconstructed from a number of given projections have been obtained by J. Matoušek, A. Přívětivý, and P. S̆kovron̆ [37] (related results can also be found in [38], [39], and [40]). In general, the size of a finite set is properly defined by its cardinality. However, in the reconstruction problem one usually has to recover discrete images inside a bounded grid by means of their tomographic data. Thus, it seems meaningful to measure the size of a finite set by the size $m \times n$ of the smallest box $\mathcal{A}=\left\{(i, j) \in \mathbb{Z}^{2}: 0 \leq i<m, 0 \leq j<n\right\}$ containing the set. Here we shall adopt this latter approach so that "minimal" refers to the rectangular grid containing the $S$-bad configuration.

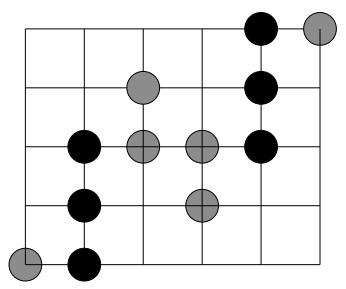

(a)

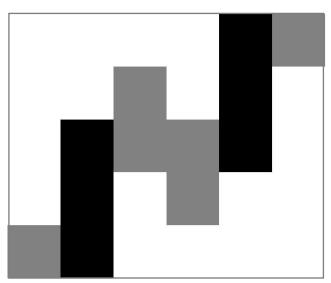

(b)

Fig. 2: (a) A bad configuration associated to $S=$ $\{(1,0),(1,1),(2,1),(1,2)\}$, with $Z, W$ formed by grey and black points respectively. (b) Digital representation of the bad configuration.

Definition 2 An $S$-(weakly) bad configuration $(Z, W)$ contained in a finite rectangular lattice grid $\mathcal{A}=\{(i, j) \in$ $\left.\mathbb{Z}^{2}: 0 \leq i<m, 0 \leq j<n\right\}$ is said to be minimal if no rectangular lattice grid $\mathcal{A}^{\prime}$ exists such that $(Z, W) \subset \mathcal{A}^{\prime} \subset \mathcal{A}$.

As a summary, as ghosts are responsible for ambiguous reconstructions, their absence implies uniqueness, which represents the goal for an accurate reconstruction. In this context, we focus on minimal ghosts and their construction in order to avoid them towards uniqueness.

Besides, minimal ghosts (see for instance [41-43]) are largely employed in digital imaging computing as an entanglement image/anti-image, which can be used to achieve forward error-correction in redundant data transmission schemes.

In a different scenario, ghosts are employed to recover the exact invertibility of the prime-sized array Finite Radon Transform (FRT) in the case of missing data (see [44]). From the rich literature focusing on ghosts (see for instance [41-43], and the related bibliography) it comes out that some of their fascinating geometric properties are still unclear, so that any new step throwing light on them would be appreciable.

From an applicative point of view, understanding how added ghosts into real image data can be managed could be useful for a comparison among different reconstruction algorithms according to a same idea as in [13]. In particular, adding binary ghosts causes the weakest 
perturbation of the original data. It follows that explicit procedures for reducing complex ghosts artifacts to simpler ones would be highly desirable. In this context our approach shows a theoretical model for treating ghost artifacts from an algebraic point of view which allows easy constructions of ghost artifacts, otherwise inaccessible in a direct geometric investigation. It turns out that any ghost artifact origins from a simpler one, which, however, might be hidden under a more complex structure (modeled as colored, or grey-scaled pixels), as a result of several overlapping and translational procedures. As far as we know, our strategy represents a first contribution in reducing such a "structured" artifact to a "simple" binary ghost, and without changing the set of projections.

\subsection{Methods and paper organization}

To prove our results, we apply the following method. We introduce a polynomial $F_{S}(x, y)$, related to the set $S$ of directions. Then, it turns out that finding an $S$-bad configuration is in fact equivalent to finding multiples of $F_{S}$ having only coefficients in the set $\{-1,0,1\}$. Note that in general, finding such multiples of polynomials is an interesting, but rather deep problem (see e.g. the paper [1], and the references there). However, by using certain special properties of the polynomial $F_{S}$, it is hopeful to give some kind of solution to the problem. In particular, in Section 3 we determine a general theoretical upper bound for the size of the smallest bad configurations associated to a given set $S$ of $k$ lattice directions. In Section 4, we prove that in the special but interesting case of four directions, the upper bound can be considerably improved by the aid of a certain reduction method. Finally, in Section 5, we propose an algorithm based on this reduction method which permits to remove the multiplicities of a given input polynomial. In the context of tomographically reconstructed digital images, it leads to a filtering, or reduction, procedure which return any binary ghost.

As our proofs and methods are somewhat technical at certain points, we provide several examples to help a better understanding.

\section{Notation, definitions and background}

Let $a, b \in \mathbb{Z}$ with $\operatorname{gcd}(a, b)=1$ and $a \geq 0$, with the further assumption that $b=1$ if $a=0$. We call $(a, b)$ a lattice direction. By lines with direction $(a, b) \in \mathbb{Z}^{2}$ we mean lattice lines defined in the $x, y$ plane by equations of the form $a y=b x+t$, where $t \in \mathbb{Z}$. We refer to a finite subset of $\mathbb{Z}^{2}$ as a lattice set.
Let $S=\left\{\left(a_{i}, b_{i}\right)\right\}_{i=1}^{k}$ be a set of directions. For $i=$ $1, \ldots, k$ put

$$
f_{\left(a_{i}, b_{i}\right)}(x, y)= \begin{cases}x^{a_{i}} y^{b_{i}}-1 & \text { if } a_{i} \neq 0, b_{i}>0 \\ x^{a_{i}}-y^{-b_{i}} & \text { if } a_{i} \neq 0, b_{i}<0 \\ x-1 & \text { if } a_{i}=1, b_{i}=0 \\ y-1 & \text { if } a_{i}=0, b_{i}=1\end{cases}
$$

and for $1 \leq j \leq k$ let

$$
F_{S}^{(j)}(x, y)=\prod_{i=1}^{j} f_{\left(a_{i}, b_{i}\right)}(x, y) .
$$

Further, we will simply use $F_{S}(x, y)$ to denote $F_{S}^{(k)}(x, y)$. Let $\mathcal{A}=\left\{(i, j) \in \mathbb{Z}^{2}: 0 \leq i<m, 0 \leq j<n\right\}$ be a finite grid. For any function $g: \mathcal{A} \rightarrow \mathbb{Z}$, its generating function is the polynomial defined by

$$
G_{g}(x, y)=\sum_{(i, j) \in A} g(i, j) x^{i} y^{j} .
$$

Conversely, we say that the function $g$ is generated by a polynomial $P(x, y)$ if $P(x, y)=G_{g}(x, y)$. Further, the line sum of $g$ along the lattice line with equation $a y=$ $b x+t$ is defined as $\sum_{a j=b i+t} g(i, j)$. It is easy to see that $f$ generated by $F_{S}(x, y)$ has zero line sums along the lines taken in the directions in $S$. Moreover, $f$ vanishes outside $\mathcal{A}$ if and only if the set $S=\left\{\left(a_{i}, b_{i}\right)\right\}_{i=1}^{k}$ of $k$ lattice directions is valid for $\mathcal{A}$, namely if

$$
\sum_{i=1}^{k} a_{i}<m, \quad \sum_{i=1}^{k}\left|b_{i}\right|<n .
$$

\subsection{Geometric interpretation of ghost reduction}

A monomial $t x^{a} y^{b} \in \mathbb{Z}[x, y]$ can be associated to the lattice point $\mathbf{p}=(a, b)$, together with the weight $t$. If $|t|>1$ we say that $\mathbf{p}$ is a multiple point and $|t|$ is its multiplicity. Therefore, a generating function corresponds, geometrically, to a lattice set whose points have associated multiplicities. For a polynomial $P(x, y)$, we denote by $P^{+}(x, y)$ (resp. $\left.P^{-}(x, y)\right)$ the polynomial formed by the monomials of $P(x, y)$ having positive (resp. negative) coefficients. The lattice sets consisting of the lattice points (counted with their multiplicities) which correspond to $P(x, y), P^{+}(x, y), P^{-}(x, y)$ are here denoted by $P, P^{+}$and $P^{-}$, respectively. We also refer to the points in $P^{+}$and $P^{-}$as points with positive and negative signs, respectively.

From the geometric point of view, the pair of sets $\left(P^{+}, P^{-}\right)$, associated to a polynomial $P(x, y)=G_{g}(x, y)$ ( $g$ being a function with zero line sums along the lines 
taken in all the directions in $S$ ) is a $S$-weakly bad configuration (or a complex ghost with respect to $S$ ), otherwise a $S$-bad configuration (or a binary ghost with respect to $S$ ) if its coefficients all belong to $\{-1,0,1\}$. As we mentioned already, these notions play a crucial role in investigating uniqueness problems, since any set $S$ of directions is a set of uniqueness if and only if it has no bad configurations (or binary ghosts).

In what follows polynomial multiplication is exploited to reduce a weakly bad configuration to a bad configuration (or a complex ghosts to a binary ghost), without changing the set $S$ of projection directions. This reflects geometrically in several overlapping and translational procedures. In particular, when the generating function of a (weakly) bad configuration is multiplied by $x^{a} y^{b}$, then this is the same as translating the (weakly) bad configuration by $(a, b)$. Also, when multiplying by $\left(x^{a} y^{b}-1\right)$, it is equivalent to subtracting the initial (weakly) bad configuration from its shifting by $(a, b)$. If a translation by vector $(a, b)$ causes points to overlap, then we could always cancel such overlapping by translating $r$ times of $(a, b)$, for some positive or negative integer $r$. However, since many different multiplicities are usually involved, the hard trick is to find that combination of $r$ values that allows the removal of all multiplicities. We refer the reader to Subsection 4.2 for further geometric insights on the reduction procedure of complex ghosts.

\subsection{Previous results employed}

In this paper we will make use frequently of some results from [28], in particular Lemma 6 and Proposition 1 , so, below, we will briefly recall the statements. For $u=(h, k) \in \mathbb{Z}^{2}$, let $f_{-}^{u}: \mathbb{Z}^{2} \rightarrow \mathbb{Z}, f_{+}^{u}: \mathbb{Z}^{2} \rightarrow \mathbb{Z}$ be the maps whose generating functions are $G_{f_{-}^{u}}(x, y)=$ $\left(x^{h} y^{k}-1\right) F_{S}(x, y)$ and $G_{f_{+}^{u}}(x, y)=\left(x^{h} y^{k}+1\right) F_{S}(x, y)$, respectively. Notice that for $h<0$ or $k<0, G_{f_{-}^{u}}, G_{f_{+}^{u}}$ are rational functions, which can be mapped to polynomials by integer translations. In [28] it was shown that, for $|S|=4$, the unique situations where $F_{S}$ has some coefficient outside $\{-1,0,1\}$ correspond, precisely, to the choices $u_{1}+u_{2}+u_{3}=u_{4}$ or $u_{1}+u_{2}-u_{3}=u_{4}$. We represent $S$ as $S=S_{1} \cup S_{2}$, where $S_{1}=\left\{u_{1}, u_{2}, u_{3}\right\}, S_{2}=$ $\left\{u_{4}\right\}$ in the first case, and $S_{1}=\left\{u_{1}, u_{2}\right\}, S_{2}=\left\{u_{3}, u_{4}\right\}$ in the second case. Also, we define the set $S_{1}-S_{2}$ as follows

$$
S_{1}-S_{2}=\left\{ \pm\left(u_{i}-u_{j}\right), u_{i} \in S_{1}, u_{j} \in S_{2}\right\} .
$$

Lemma 1 (Lemma 6 in [28]) Let $S=\left\{u_{1}, u_{2}, u_{3}, u_{4}\right\}$ be a set of four lattice directions, such that $u_{4}=u_{1}+$ $u_{2} \pm u_{3}$. Then the following hold
1. $\left|f_{-}^{u}\right| \leq 1$ if and only if $u=0$, or $u \in\left(S_{1}-S_{2}\right)$ and $u_{4}=u_{1}+u_{2}+u_{3}$, or $u \in\left(S_{1}-S_{2}\right) \cup\left\{ \pm\left(u_{1}+u_{2}\right)\right\}$ and $u_{4}=u_{1}+u_{2}-u_{3}$.

2. $\left|f_{+}^{u}\right| \leq 1$ if and only if $u \in S$ or $-u \in S$.

Let $g: \mathbb{Z}^{2} \rightarrow \mathbb{Z}$ be a non trivial function whose generating function is defined by $G_{g}(x, y)=P(x, y) F_{S}(x, y)$, for some polynomial $P(x, y)$, consisting of $r$ monomials. For $h \in\{1, \ldots, r\}$, let $g_{h}: \mathbb{Z}^{2} \rightarrow \mathbb{Z}$ be the function whose generating function is determined by multiplying $F_{S}(x, y)$ by the first (with respect to the given order in $P(x, y)) h$ monomials of $P(x, y)$.

Let

$$
Q_{h}(S)=\left\{(i, j) \in \mathbb{Z}^{2}: g_{h}(i, j) \neq 0\right\}
$$

and

$$
\begin{aligned}
& \mathcal{P}_{h}=\left\{(i, j) \in Q_{h}(S): g_{h}(i, j)>0\right\} \\
& \mathcal{N}_{h}=\left\{(i, j) \in Q_{h}(S): g_{h}(i, j)<0\right\}
\end{aligned}
$$

In particular $Q_{0}(S)=\left(F_{S}^{+}, F_{S}^{-}\right), \mathcal{P}_{0}=F_{S}^{+}$, and $\mathcal{N}_{0}=F_{S}^{-}$. Let $M_{h}$ denote the set of multiple points of $Q_{h}(S)$, and let $M_{h}^{+}, M_{h}^{-}$be the subsets of $M_{h}$ formed by the points of $M_{h}$ belonging to $\mathcal{P}_{h}$ and $\mathcal{N}_{h}$, respectively. In particular $M_{0}=\{w\}$, namely the multiple point of $Q(S)$. Moreover, for any pair of sets $X, Y$, we define $X-Y=Y-X=\{ \pm(x-y): x \in X, y \in Y\}$.

Proposition 1 (Proposition 1 in [28]) ${ }^{1}$ Let $S=$ $\left\{u_{1}, u_{2}, u_{3}, u_{1}+u_{2} \pm u_{3}\right\}$ be a set of four lattice directions. Let $g: \mathbb{Z}^{2} \rightarrow \mathbb{Z}$ be a non trivial function whose generating function is defined by

$$
G_{g}(x, y)=\sum_{t=1}^{r} \delta(t) x^{i(t)} y^{j(t)} F_{S}(x, y),
$$

where $\delta(t)= \pm 1$. For each $h \in\{0, \ldots, r-1\}$, consider the monomial $\delta(h+1) x^{i(h+1)} y^{j(h+1)}$. Suppose that $\delta(h+$ $1)=1$ and the following conditions hold

1. If $(c, d) \in M_{h}^{+}$then $(i(h+1), j(h+1))=(c, d)-$ $(e, f)$, with $(e, f) \in \mathcal{N}$.

2. If $(c, d) \in M_{h}^{-}$then $(i(h+1), j(h+1))=(c, d)-$ $(e, f)$, with $(e, f) \in \mathcal{P}$.

3. $(i(h+1), j(h+1)) \notin\left(\mathcal{P}_{h}-\mathcal{P}\right) \cup\left(\mathcal{N}_{h}-\mathcal{N}\right)$.

4. $(i(h+1), j(h+1))+w \in \mathcal{N}_{h}$ if $w \in M_{0}^{+},(i(h+$ 1), $j(h+1))+w \in \mathcal{P}_{h}$ if $w \in M_{0}^{-}$.

Then $M_{h+1} \subseteq M_{h}$.

1 In [28, Proposition 1] the pair $(i(h+1), i(h+1))$ must be replaced by $(i(h+1), j(h+1))$, and $h \in\{1, \ldots, r\}$ with $h \in\{0, \ldots, r-1\}$. 


\section{Minimal bad configurations}

\section{$3.1 k$ directions}

In this section we derive bounds for the size of a maximal grid $\mathcal{A}=\left\{(i, j) \in \mathbb{Z}^{2}: 0 \leq i<m, 0 \leq j<n\right\}$ which does not contain $S$-bad configurations. It may happen that these bounds are not optimal, as it is shown by Example 1 (see also Remark 1).

Theorem 1 Let $S=\left\{\left(a_{i}, b_{i}\right)\right\}_{i=1}^{k}$ be a set of directions, where $0 \leq a_{1} \leq \cdots \leq a_{k}$. Let $\mathcal{A}=\left\{(i, j) \in \mathbb{Z}^{2}\right.$ : $0 \leq i<m, 0 \leq j<n\}$ be a grid which does not contain $S$-bad configurations. Then $m \leq\left(2^{k+1}-1\right) a_{k}$ or $n \leq\left(2^{k+1}-1\right) B$, where $B=\max \left\{\left|b_{1}\right|, \ldots,\left|b_{k}\right|\right\}$.

In order to prove the Theorem, we shall need the following Lemma, which provides an inductive procedure, in the number of directions belonging to $S$, to construct a polynomial $G(x, y)$ having coefficients in $\{-1,0,1\}$, such that $F_{S}(x, y)$ divides $G(x, y)$.

Lemma 2 Let $S=\left\{\left(a_{i}, b_{i}\right)\right\}_{i=1}^{k}$ be a set of directions, where $0 \leq a_{1} \leq \cdots \leq a_{k}$. Then there exists a polynomial $G(x, y)=R(x, y) F_{S}(x, y)$, having coefficients in $\{-1,0,1\}$, such that $\operatorname{deg}_{x} G \leq\left(2^{k+1}-1\right) a_{k}, \operatorname{deg}_{y} G \leq$ $\left(2^{k+1}-1\right) B$, where $B=\max \left\{\left|b_{1}\right|, \ldots,\left|b_{k}\right|\right\}$.

Proof Take $a_{1}^{*}:=a_{1}$ and $b_{1}^{*}:=b_{1}$, and let

$$
\begin{gathered}
G_{1}^{+}(x, y)= \begin{cases}x^{a_{1}} y^{b_{1}} & \text { if } a_{1} \neq 0, b_{1}>0, \\
x^{a_{1}} & \text { if } a_{1} \neq 0, b_{1}<0, \\
x & \text { if } a_{1}=1, b_{1}=0, \\
1 & \text { if } a_{1}=0, b_{1}=1,\end{cases} \\
G_{1}^{-}(x, y)= \begin{cases}1 & \text { if } a_{1} \neq 0, b_{1}>0, \\
y^{-b_{1}} & \text { if } a_{1} \neq 0, b_{1}<0, \\
1 & \text { if } a_{1}=1, b_{1}=0, \\
y & \text { if } a_{1}=0, b_{1}=1 .\end{cases}
\end{gathered}
$$

Observe that the above polynomials have only coefficients in $\{0,1\}$. Thus putting $G_{1}(x, y)=G_{1}^{+}(x, y)-$ $G_{1}^{-}(x, y), G_{1}(x, y)$ has only coefficients in $\{-1,0,1\}$, and is obviously divisible by $F_{S}^{(1)}$. Moreover, the degrees of $G_{1}^{+}(x, y)$ and $G_{1}^{-}(x, y)$ in $x$ are at most $a_{1}^{*}=a_{1}$.

Assume now that for some $j$ with $1 \leq j<k$, the integers $a_{j}^{*}, b_{j}^{*}$ and polynomials $G_{j}^{+}(x, y), G_{j}^{-}(x, y)$ are already defined, so that both $G_{j}^{+}(x, y), G_{j}^{-}(x, y)$ have only 0,1 -coefficients, the degrees of these polynomials are at most $\left(2^{j+1}-1\right) a_{j}$ in $x$, and the polynomial

$$
G_{j}(x, y):=G_{j}^{+}(x, y)-G_{j}^{-}(x, y)
$$

is divisible by $F_{S}^{(j)}(x, y)$. Put $a_{j+1}^{*}=2^{j+1} a_{j+1}$ and $b_{j+1}^{*}=2^{j+1} b_{j+1}$. Note that $a_{j+1}>0$, and let

$$
\begin{aligned}
g_{j+1}^{+}(x, y) & = \begin{cases}x^{a_{j+1}^{*}} y^{b_{j+1}^{*}} & \text { if } b_{j+1}>0, \\
x^{a_{j+1}^{*}} & \text { if } b_{j+1}<0, \\
x^{a_{j+1}^{*}} & \text { if } a_{j+1}=1, b_{j+1}=0,\end{cases} \\
g_{j+1}^{-}(x, y) & = \begin{cases}1 & \text { if } b_{j+1}>0, \\
y^{-b_{j+1}^{*}} & \text { if } b_{j+1}<0, \\
1 & \text { if } a_{j+1}=1, b_{j+1}=0 .\end{cases}
\end{aligned}
$$

Set $G_{j+1}^{+}(x, y)=g_{j+1}^{+}(x, y) \cdot G_{j}^{+}(x, y)+g_{j+1}^{-}(x, y) \cdot G_{j}^{-}(x, y)$ and $G_{j+1}^{-}(x, y)=g_{j+1}^{-}(x, y) \cdot G_{j}^{+}(x, y)+g_{j+1}^{+}(x, y)$. $G_{j}^{-}(x, y)$. Observe that by the induction hypothesis and the definition of $a_{j+1}^{*}$, both $G_{j+1}^{+}(x, y)$ and $G_{j+1}^{-}(x, y)$ have only 0,1 -coefficients. Further, we easily get that the degrees of $G_{j}^{+}(x, y)$ and $G_{j}^{-}(x, y)$ are at most $\left(2^{j+1}-\right.$ 1) $a_{j+1}$ in $x$. Putting $G_{j+1}(x, y)=G_{j+1}^{+}(x, y)-G_{j+1}^{-}(x, y)$, we have

$$
G_{j+1}(x, y)=\left(g_{j+1}^{+}(x, y)-g_{j+1}^{-}(x, y)\right)\left(G_{j}^{+}(x, y)-G_{j}^{-}(x, y)\right) .
$$

The first factor on the right hand side is obviously divisible by $f_{\left(a_{j+1}, b_{j+1}\right)}(x, y)$, while the second one by $F_{S}^{(j)}(x, y)$ by induction. Hence we also obtain that $F_{S}^{(j+1)}(x, y)$ divides $G_{j+1}(x, y)$.

Let $G(x, y)=G_{k}(x, y)$. By induction we get that $G(x, y)$ has $-1,0,1$-coefficients, $F_{S}(x, y)$ divides $G(x, y)$ and the degree of $G(x, y)$ in $x$ is at most $\left(2^{k+1}-1\right) a_{k}$. Since it is easy to check that the degree of $G(x, y)$ is at most $\left(2^{k+1}-1\right) B$ in $y$, our claim follows.

Notice that the proof is constructive so that it designs the steps for generating ghosts.

Remark 1 The provided upper bounds on $\operatorname{deg}_{x} G(x, y)$ and $\operatorname{deg}_{y} G(x, y)$, depend only on the directions in $S$. However, we could get better results by taking advantage of the previous steps in the selection of $a_{j+1}^{*}$ and $b_{j+1}^{*}$. For instance, the above method can be improved in the following way. At the $(j+1)$-th step instead of taking $a_{j+1}^{*}=2^{j+1} a_{j+1}$ and $b_{j+1}^{*}=2^{j+1} b_{j+1}$, find the smallest positive integer $t_{j+1}$ such that either $t_{j+1} a_{j+1}>$ $\sum_{i=1}^{j} a_{i}^{*}$ or $t_{j+1}\left|b_{j+1}\right|>\sum_{i=1}^{j}\left|b_{i}^{*}\right|$ holds. Then letting $a_{j+1}^{*}=$ $t_{j+1} a_{j+1}$ and $b_{j+1}^{*}=t_{j+1} b_{j+1}$, one can easily check that the resulting polynomial $G(x, y)=G_{k}(x, y)$ has the same properties as before.

Proof (of Theorem 1) There is a one-to-one correspondence between $S$-bad configurations $(Z, W)$ contained in $\mathcal{A}$ and non-trivial functions $h: \mathcal{A} \rightarrow\{-1,0,1\}$ having zero line sums along the lines corresponding to the directions in $S$, by defining $h(z)=1$ if $z \in Z, h(z)=-1$ if $z \in W$, and $h(z)=0$ otherwise. Hajdu and Tijdeman 
showed that if $h: \mathcal{A} \rightarrow \mathbb{Z}$ has zero line sums along the lines taken in the directions in $S$, then $F_{S}(x, y)$ divides $G_{h}(x, y)$ over $\mathbb{Z}$ ([25, Lemma 3.1]). Therefore, if $m>\left(2^{k+1}-1\right) a_{k}$ and $n>\left(2^{k+1}-1\right) B$ then the grid $\mathcal{A}$ contains the $S$-bad configuration $\left(G^{+}, G^{-}\right)$associated to the polynomial $G(x, y)$ defined in Lemma 2 .

Theorem 1 states that if, on the contrary, $m>$ $\left(2^{k+1}-1\right) a_{k}$ and $n>\left(2^{k+1}-1\right) B$, ghosts may be hidden in $\mathcal{A}$.

Example 1 Assume $S=\{(0,1),(1,0),(1,2),(2,1)\}$, so that $k=4, a_{1}=0, a_{2}=a_{3}=1, a_{4}=2$, and $b_{1}=$ $1, b_{2}=0, b_{3}=2, b_{4}=1$ (alternatively we could choose $\left.b_{1}=1, b_{2}=2, b_{3}=0, b_{4}=1\right)$. The inductive steps provide the following results.

- $j=1 a_{1}^{*}=a_{1}=0, b_{1}^{*}=b_{1}=1, G_{1}^{+}(x, y)=1$, $G_{1}^{-}(x, y)=y$,

$$
G_{1}(x, y)=1-y \text {. }
$$

- $j=2 a_{2}^{*}=4, b_{2}^{*}=0, g_{2}^{+}(x, y)=x^{4}, g_{2}^{-}(x, y)=1$,

$$
\begin{aligned}
& G_{2}^{+}(x, y)=x^{4} \cdot 1+1 \cdot y=x^{4}+y \\
& G_{2}^{-}(x, y)=1 \cdot 1+x^{4} \cdot y=1+x^{4} y \\
& G_{2}(x, y)=\left(x^{4}-1\right)(1-y) .
\end{aligned}
$$

- $j=3 a_{3}^{*}=8, b_{3}^{*}=16, g_{3}^{+}(x, y)=x^{8} y^{16}, g_{3}^{-}(x, y)=1$,

$G_{3}^{+}(x, y)=$

$x^{8} y^{16} \cdot\left(x^{4}+y\right)+1 \cdot\left(1+x^{4} y\right)=x^{12} y^{16}+x^{8} y^{17}+x^{4} y+1$

$G_{3}^{-}(x, y)=$

$x^{8} y^{16} \cdot\left(1+x^{4} y\right)+1 \cdot\left(x^{4}+y\right)=x^{8} y^{16}+x^{12} y^{17}+x^{4}+y$

$G_{3}(x, y)=\left(x^{8} y^{16}-1\right)\left(x^{4}-1\right)(1-y)$.

- $j=k=4 a_{4}^{*}=32, b_{4}^{*}=16, g_{4}^{+}(x, y)=x^{32} y^{16}$, $g_{4}^{-}(x, y)=1$.

$G_{4}^{+}(x, y)=x^{32} y^{16} \cdot\left(x^{12} y^{16}+x^{8} y^{17}+x^{4} y+1\right)$

$+1 \cdot\left(x^{8} y^{16}+x^{12} y^{17}+x^{4}+y\right)=$

$=x^{44} y^{33}+x^{40} y^{33}+x^{36} y^{17}+x^{32} y^{16}$

$+x^{8} y^{16}+x^{12} y^{17}+x^{4}+y$

$G_{4}^{-}(x, y)=x^{32} y^{16} \cdot\left(x^{8} y^{16}+x^{12} y^{17}+x^{4}+y\right)$

$+1 \cdot\left(x^{12} y^{16}+x^{8} y^{17}+x^{4} y+1\right)=$

$=x^{44} y^{33}+x^{40} y^{32}+x^{36} y^{16}$

$+x^{32} y^{17}+x^{12} y^{17}+x^{8} y^{17}+x^{4} y+1$

$G(x, y)=G_{4}(x, y)=$

$=\left(x^{32} y^{16}-1\right)\left(x^{8} y^{16}-1\right)\left(x^{4}-1\right)(1-y)=$

$=-x^{44} y^{33}+x^{44} y^{32}+x^{40} y^{33}-x^{40} y^{32}+$

$+x^{36} y^{17}-x^{36} y^{16}-x^{32} y^{17}+x^{32} y^{16}+$

$+x^{12} y^{17}-x^{12} y^{16}-x^{8} y^{17}+x^{8} y^{16}-x^{4} y+x^{4}+y-1$.
Note that $\operatorname{deg}_{x} G(x, y)$ and $\operatorname{deg}_{y} G(x, y)$ satisfy the upper bound in Theorem 2 with the strict inequality. These can be considerably lowered as described in Remark 1. In fact, we get $t_{2}=1, t_{3}=1, t_{4}=2$, which gives $a_{2}^{*}=1, b_{2}^{*}=0, a_{3}^{*}=1, b_{3}^{*}=2, a_{4}^{*}=4, b_{4}^{*}=2$, and consequently

$$
\begin{gathered}
G(x, y)=G_{4}(x, y)= \\
=(1-y)(x-1)\left(x y^{2}-1\right)\left(x^{4} y^{2}-1\right)= \\
=-x^{6} y^{5}+x^{5} y^{5}+x^{6} y^{4}-x^{5} y^{4}+x^{5} y^{3}-x^{4} y^{3}+x^{2} y^{3} \\
-x y^{3}-x^{5} y^{2}+x^{4} y^{2}-x^{2} y^{2}+x y^{2}-x y+y+x-1 .
\end{gathered}
$$

\subsection{Four directions}

From an algebraic point of view, the focus of the previous section is the lowering of the weights of the multiple points belonging to a basic weakly bad configuration which corresponds to the set of lattice points associated to the polynomial $F_{S}(x, y)$ (corresponding to a set $S$ of $k$ different lattice directions, where $k$ is arbitrarily chosen).

We now observe that the procedure described in the proof of Lemma 2 provides the required polynomial $G(x, y)$ having coefficients in $\{-1,0,1\}$ without any check on the starting polynomial $F_{S}(x, y)$. This causes the degree of $G(x, y)$ to be high compared to the degree of $F_{S}(x, y)$. Note that $F_{S}(x, y)$ could have no multiple points at all, so that the problem would be trivial. Thus, some analysis of the coefficients of $F_{S}(x, y)$ not belonging to $\{-1,0,1\}$ could provide useful information for bounding the degree of a possible multiple polynomial with coefficients in $\{-1,0,1\}$. For instance, the set $S$ as in Example 1 provides the following polynomial:

$$
\begin{aligned}
& F_{S}(x, y)=x^{4} y^{4}-x^{4} y^{3}-x^{3} y^{4}+x^{3} y^{3}-x^{3} y^{2}+x^{3} y- \\
& -x^{2} y^{3}+2 x^{2} y^{2}-x^{2} y+x y^{3}-x y^{2}+x y-x-y+1
\end{aligned}
$$

where we have just one coefficient not belonging to $\{-1,0,1\}$. Such a multiplicity can be removed as required simply taking $G(x, y)=\left(x^{h} y^{k}+1\right) F_{S}(x, y)$, with $(h, k) \in S$ (alternatively also $G(x, y)=\left(x^{h} y^{k}-1\right) F_{S}(x, y)$ for some $(h, k)$ suitably selected), as shown in [28, Lemma $6]$. For instance, we can take $(h, k)=(1,0)$, so that

$G(x, y)=(x+1) F_{S}(x, y)=x^{5} y^{4}-x^{5} y^{3}-x^{4} y^{2}+x^{4} y-$ $-x^{3} y^{4}+x^{3} y^{2}+x^{2} y^{2}-x^{2}+x y^{3}-x y^{2}-y+1$,

and the degrees in $x$ and $y$ are even lower than those in (2). 
Starting from the above remarks, we now approach the problem presented in the previous section in a different way. Instead of an arbitrary number of directions in the set $S$, we fix the cardinality of $S$, and consider the polynomial $H(x, y) F_{S}(x, y)$, for a given polynomial $H(x, y)$. Then, we look for possible polynomials $K(x, y)$, such that $G(x, y)=(H(x, y)+K(x, y)) F_{S}(x, y)$ has all the coefficients in $\{-1,0,1\}$. In particular, we assume that $S=\left\{u_{1}, u_{2}, u_{3}, u_{4}\right\}$, with $u_{1}+u_{2}+u_{3}=u_{4}$ or $u_{1}+u_{2}-u_{3}=u_{4}$. Motivation for this choice relies on the fact that by [30, Theorem 2.4], less than four directions are never sufficient to distinguish all the subsets of a given grid $\mathcal{A}$, and, for $|S|=4$, the unique situations where $F_{S}$ represents a weakly bad configuration correspond, precisely, to the choices $u_{1}+u_{2}+u_{3}=u_{4}$ or $u_{1}+u_{2}-u_{3}=u_{4}$ (see [28]). Therefore, for a set of four directions, these are the unique cases where the problem is not trivial.

We first note that if $H(x, y)=1$, then a possible solution to the above problem is provided by $K(x, y)=$ $R(x, y)-1$, where $R(x, y)$ is obtained according to Theorem 2. However, if $S=\left\{u_{1}, u_{2}, u_{3}, u_{4}\right\}$, with $u_{1}+u_{2} \pm$ $u_{3}=u_{4}$, we can find a significant improvement. It is shown by the following result, which follows from [28, Lemma 6].

Proposition 2 Let $S=\left\{u_{i}=\left(a_{i}, b_{i}\right)\right\}_{i=1}^{4}$ be a set of four directions, where $u_{1}+u_{2} \pm u_{3}=u_{4}$. Then, for each $i \in\{1,2,3,4\}$ the polynomial

$G_{i}(x, y)= \begin{cases}\left(x^{a_{i}} y^{b_{i}}+1\right) F_{S}(x, y), & \text { if } b_{i} \geq 0 \\ \left(x^{a_{i}}+y^{\left|b_{i}\right|}\right) F_{S}(x, y), & \text { if } b_{i}<0\end{cases}$

has coefficients in $\{-1,0,1\}$.

This result allows us to improve the bounds obtained in Theorem 1 on the size of a maximal grid which does not contain $S$-bad configurations.

Corollary 1 Let $S=\left\{u_{i}=\left(a_{i}, b_{i}\right)\right\}_{i=1}^{4}$ be a set of four directions, where $u_{1}+u_{2} \pm u_{3}=u_{4}$. Let $\mathcal{A}=\{(i, j) \in$ $\left.\mathbb{Z}^{2}: 0 \leq i<m, 0 \leq j<n\right\}$ be a grid which does not contain $S$-bad configurations. Then $m \leq a_{i}+\sum_{k=1}^{4} a_{k}$ or $n \leq\left|b_{i}\right|+\sum_{k=1}^{4}\left|b_{k}\right|$, for each $i \in\{1,2,3,4\}$.

Proof Note that the polynomial $G_{i}(x, y)$ defined in (3) has degrees $\operatorname{deg}_{x} G_{i}(x, y)=a_{i}+\sum_{k=1}^{4} a_{k}, \operatorname{deg}_{y} G_{i}(x, y)=$ $\left|b_{i}\right|+\sum_{k=1}^{4}\left|b_{k}\right|$, for each $i \in\{1,2,3,4\}$. Suppose that $m>a_{i}+\sum_{k=1}^{4} a_{k}$ and $n>\left|b_{i}\right|+\sum_{k=1}^{4}\left|b_{k}\right|$, for each $i \in\{1,2,3,4\}$. Then the grid $\mathcal{A}$ contains the $S$-bad configuration $\left(G_{i}^{+}, G_{i}^{-}\right)$associated to the polynomial $G_{i}(x, y)$ defined in $(3)$.

\section{From overlapping weakly bad configurations to binary ghosts}

We now address the problem for an arbitrary polynomial $H(x, y)$ and $S=\left\{u_{1}, u_{2}, u_{3}, u_{4}\right\}$, where $u_{1}+u_{2} \pm$ $u_{3}=u_{4}$.

Problem 1 Given a polynomial $H(x, y)$ with integer coefficients, and $S=\left\{u_{1}, u_{2}, u_{3}, u_{4}\right\}$, where $u_{1}+u_{2} \pm$ $u_{3}=u_{4}$, find a polynomial $K(x, y)$ such that $G(x, y)=$ $(H(x, y)+K(x, y)) F_{S}(x, y)$ has all the coefficients belonging to $\{-1,0,1\}$.

The configuration $P$ associated to a given polynomial $P(x, y)=H(x, y) F_{S}(x, y)$ with integer coefficients can be seen as an original bad configuration which has been modified as a result of several overlapping and translational procedures. Feasible solutions to Problem 1 can be obtained by providing a filtering (or reduction) procedure, which can be assumed to be additive as well, which returns a bad configuration as an output. This corresponds to the construction of a new polynomial

$$
K(x, y) F_{S}(x, y)
$$

where $K(x, y)$ is a polynomial with only $\{0,1\}$ coefficients, such that $G(x, y)=(H(x, y)+K(x, y)) F_{S}(x, y)$ has all its coefficients belonging to $\{-1,0,1\}$.

If a solution exists, then the polynomial

$$
P(x, y)=H(x, y) F_{S}(x, y)
$$

is said to be reducible, and $G(x, y)$ is said to be a reduction of $P(x, y)$. The corresponding lattice bad configuration is said to be the binary ghost associated to the original (corrupted) image.

\subsection{Algebraic approach to a reduction procedure}

From a geometric point of view, the set $P$ associated to the polynomial $P(x, y)=H(x, y) F_{S}(x, y)$ corresponds to mutually overlapping translations of the $S$-weakly bad configuration $F_{S}$ associated to $F_{S}(x, y)$, where each translation along a vector $(a, b)$ corresponds to a monomial $k x^{a} y^{b}$ in $H(x, y)$. We shall find the polynomial $K(x, y)$ by considering all the possible monomials $k x^{a} y^{b}$ which remove one or more multiple points in $P$ without adding new multiple points, until a resulting set is obtained with no multiple points. Our method bases on Proposition 1, which we now restate in a slightly modified version for a better understanding.

To this, we can assume $b \geq 0$ for all $(a, b) \in S$, so that $F_{S}^{+}$consists of the points

$\mathbf{0}, u_{1}+u_{2}, u_{1}+u_{3}, u_{1}+u_{4}, u_{2}+u_{3}$,

$u_{2}+u_{4}, \quad u_{3}+u_{4}, \quad u_{1}+u_{2}+u_{3}+u_{4}$, 
and $F_{S}^{-}$consists of

$$
\begin{aligned}
& u_{1}, \quad u_{2}, \quad u_{3}, \quad u_{4}, \\
& u_{1}+u_{2}+u_{3}, u_{1}+u_{2}+u_{4}, \\
& u_{1}+u_{3}+u_{4}, u_{2}+u_{3}+u_{4},
\end{aligned}
$$

where the points are not all necessarily distinct.

Note that the choice of $S$ implies that $F_{S}$ has just a single double point $\mathbf{w}=(1 / 2)\left(u_{1}+u_{2}+u_{3}+u_{4}\right)$.

Moreover, if $b<0$ for some $(a, b) \in S$, then $F_{S}^{+}$and $F_{S}^{-}$ consist of the previous sets of points translated by the vector $(0,-b)$, and the following arguments still hold with simple modifications.

We also assume, for simplicity, that all the monomials in $H(x, y)$ have positive coefficients. Let $M_{P}$ denote the set of multiple points of $P$, and let $M_{P}^{+}, M_{P}^{-}$be the sets of multiple points with positive and negative signs, respectively. Assume $(c, d) \in M_{P}$, and denote by $k_{P}(c, d)$ its multiplicity. Consider a vector $(a, b)$ such that the following conditions hold:

(A) If $(c, d) \in M_{P}^{+}\left(M_{P}^{-}\right.$, respectively) then $(a, b)=$ $(c, d)-(e, f)$, for some $(e, f) \in F_{S}^{-}\left(F_{S}^{+}\right.$, respectively),

(B) $(a, b) \notin\left(P^{+}-F_{S}^{+}\right) \cup\left(P^{-}-F_{S}^{-}\right)$,

(C) $(a, b)+\mathbf{w} \in P^{-}$if $\mathbf{w} \in F_{S}^{+},(a, b)+\mathbf{w} \in P^{+}$if $\mathbf{w} \in F_{S}^{-}$.

Define

$$
\begin{aligned}
& Q(x, y)=P(x, y)+x^{a} y^{b} F_{S}(x, y)= \\
& =\left(H(x, y)+x^{a} y^{b}\right) F_{S}(x, y)
\end{aligned}
$$

and denote by $M_{Q}$ the set of multiple points of $Q$. Then, by Proposition 1, the weighted lattice configuration $Q$ associated to the polynomial $Q(x, y)$ is such that $M_{Q} \subseteq M_{P}$ and $k_{Q}(c, d)<k_{P}(c, d)$, where $k_{Q}(c, d)$ denotes the multiplicity of $(c, d)$ in $Q$. Note that, in case $k_{P}(c, d)>1$, we might have $M_{Q}=M_{P}$. However, what is really ensured, is that $k_{Q}(c, d)<k_{P}(c, d)$, and no new multiple points appear in $M_{Q}$. Therefore, we can restate Proposition 1 as follows.

Proposition 3 Let $S=\left\{u_{1}, u_{2}, u_{3}, u_{1}+u_{2} \pm u_{3}\right\}$ be a set of four lattice directions. Consider a polynomial $P(x, y)=H(x, y) F_{S}(x, y)$, where $H(x, y)$ consists of monomials with positive coefficients.

Denote by $Q$ the lattice points configuration associated to $Q(x, y)=\left(H(x, y)+x^{a} y^{b}\right) F_{S}(x, y)$. If $(c, d) \in M_{P}$ and $(a, b)$ satisfies conditions $(A),(B),(C)$, then $M_{Q} \subseteq$ $M_{P}$ and $k_{Q}(c, d)<k_{P}(c, d)$.

Proof See Proposition 1 with $(i(h+1), j(h+1))=(a, b)$, $\mathcal{P}=F_{S}^{+}, \mathcal{N}=F_{S}^{-}$.

\subsection{Geometric discussion}

For a better understanding of Proposition 3, we now present a geometric interpretation of conditions $(A)$, $(B),(C)$. Let $(c, d)$ be a multiple point of $P$, for instance $(c, d) \in M_{P}^{+}$(if $(c, d) \in M_{P}^{-}$we have analogous interpretations, with the obvious changes).

- Condition $(A)$ in Proposition 3 means that the translation of $F_{S}$ along the vector $(a, b)$ moves, in particular, a negative point of $F_{S}$ to $(c, d)$, namely

$$
(a, b)+\mathbf{n}=(c, d) \text { for some } \mathbf{n} \in F_{S}^{-} .
$$

- Condition $(C)$ means that such a translation must map the double point $\mathbf{w}$ to a point of $P$ having sign different from that of $\mathbf{w}$.

- Condition $(B)$ means that the translation of any point of $F_{S}^{-}$along $(a, b)$ does not overlap to a negative point of $P$, as well as the translation of any point of $F_{S}^{+}$along $(a, b)$ does not overlap to a positive point of $P$. We can rephrase this condition as follows

$$
\begin{gathered}
(a, b)+\mathbf{n}^{\prime} \notin P^{-} \text {for all } \mathbf{n}^{\prime} \in F_{S}^{-} \\
(a, b)+\mathbf{p}^{\prime} \notin P^{+} \text {for all } \mathbf{p}^{\prime} \in F_{S}^{+}
\end{gathered}
$$

From (6) the vector $(a, b)$ must be selected among the pairs of the set

$\left\{(c, d)-\mathbf{n}^{\prime}, \mathbf{n}^{\prime} \in F_{S}^{-}\right\}$,

so that the multiple point $(c, d)$ is translated to a point of $P$ with different sign. Moreover, from (7) and (8), a selection satisfying (9) is allowed if it does not belong to the set

$$
\left(P^{-}-F_{S}^{-}\right) \cup\left(P^{+}-F_{S}^{+}\right) .
$$

\subsection{Reduction procedure}

We say that $(a, b)$ allows the reduction of $k_{P}(c, d)$ if $(a, b)$ can be selected as in Proposition 3. For a given weighted lattice set $E$, denote by $E+(a, b)$ the set of weighted lattice points obtained by translating the points in $E$ by $(a, b)$. 
Proposition 4 Let $(c, d) \in M_{P}$, and suppose that $(a, b)$ allows the reduction of $k_{P}(c, d)$. Let $Q$ be the weighted lattice configuration associated to the polynomial $Q(x, y)=$ $P(x, y)+x^{a} y^{b} F_{S}(x, y)=\left(H(x, y)+x^{a} y^{b}\right) F_{S}(x, y)$. Then we have

$k_{Q}(\mathbf{z})<k_{P}(\mathbf{z})$ for each $\mathbf{z} \in P \cap\left(F_{S}+(a, b)\right)$.

Proof Assume $(c, d) \in M_{P}^{+}$and $(a, b)$ are selected as in Proposition 3. Then $(a, b)$ belongs to the set (9) and does not belong to the set (10). By (9) there exists $\mathbf{n} \in F_{S}^{-}$such that $(a, b)+\mathbf{n}=(c, d)$. Since $(c, d)$ is a positive (multiple) point, then there exist $\mathbf{p} \in F_{S}^{+}$, and a monomial $x^{\alpha} y^{\beta}$ of $H(x, y)$, such that $(c, d)=$ $(\alpha, \beta)+\mathbf{p}$. (We recall that all the coefficients of the polynomial $H(x, y)$ are assumed to be positive).

Let us consider a point $\mathbf{z} \in P \cap\left(F_{S}+(a, b)\right)$. Then $\mathbf{z}=(a, b)+\mathbf{x}$, where $\mathbf{x} \in F_{S}$. Assume first $\mathbf{x}=\mathbf{w}$. Since $(a, b)$ allows the reduction of $k_{P}(c, d)$, then Condition $(C)$ in Proposition 3 is satisfied. This implies that the monomials in $H(x, y) F_{S}(x, y)$ and $x^{a} y^{b} F_{S}(x, y)$ corresponding to $\mathbf{z}$ have different signs, so that (11) holds.

Assume now $\mathbf{x} \neq \mathbf{w}$. Then we have

$$
\mathbf{z}=(a, b)+\mathbf{x}=(c, d)-\mathbf{n}+\mathbf{x}=(\alpha, \beta)+\mathbf{p}-\mathbf{n}+\mathbf{x} .
$$

Suppose that $\mathbf{x} \in F_{S}^{-}$. Since $\mathbf{z} \in P$ and $(\alpha, \beta) \in H$, then $\mathbf{x}+\mathbf{p}-\mathbf{n} \in F_{S}$. By (4) and (5), $\mathbf{x}+\mathbf{p}-\mathbf{n} \in F_{S}^{+}$, since the points in $F_{S}^{+}\left(F_{S}^{-}\right)$are obtained by adding an even (odd) number of elements belonging to $S$. Thus $\mathbf{z}=(\alpha, \beta)+\mathbf{p}-\mathbf{n}+\mathbf{x}$ corresponds to a monomial in $H(x, y) F_{S}(x, y)$ with positive coefficient, since all the monomials in $H(x, y)$ are supposed to have positive coefficients. Moreover, since $\mathbf{z}=(a, b)+\mathbf{x}$ with $\mathbf{x} \in$ $F_{S}^{-}$, then it corresponds to a monomial in $x^{a} y^{b} F_{S}(x, y)$ with a negative coefficient. Therefore, we have $k_{Q}(\mathbf{z})<$ $k_{P}(\mathbf{z})$. This proves (11).

If $\mathbf{x} \in F_{S}^{+}$, then (4) and (5) imply $\mathbf{x}+\mathbf{p}-\mathbf{n} \in F_{S}^{-}$. Then we can argue as above to get (11) for each $\mathbf{z} \in$ $P \cap\left(F_{S}+(a, b)\right)$.

Proposition 3 gives a sufficient condition to lower multiplicities and Proposition 4 shows that the pair $(a, b)$ can reduce simultaneously the multiplicity of several points in $M_{P}$. In general however it is possible that one choice is not sufficient to reduce all the multiplicities. In this case, to get a reduction of $P(x, y)$ we have to guarantee that two distinct allowed choices $(a, b)$ and $\left(a^{\prime}, b^{\prime}\right)$ do not produce new multiple points, that is points not contained in $M_{P}$. On this regard, we notice that condition $(\mathrm{B})$, applied to $(a, b)$ and $\left(a^{\prime}, b^{\prime}\right)$ respectively, implies that possible multiple points associated to the polynomial $P(x, y)+\left(x^{a} y^{b}+x^{a^{\prime}} y^{b^{\prime}}\right) F_{S}(x, y)$ and not contained in $M_{P}$ must come from $\left(x^{a} y^{b}+\right.$ $\left.x^{a^{\prime}} y^{b^{\prime}}\right) F_{S}(x, y)$. This happens when the translation of a point in $F_{S}^{+}$(or $F_{S}^{-}$) by $(a, b)$ and the translation of a point in $F_{S}^{+}$(or $\left.F_{S}^{-}\right)$by $\left(a^{\prime}, b^{\prime}\right)$ overlap to produce a multiple point not in $M_{P}$. This suggests the following definition.

Definition 3 Let $(c, d),\left(c^{\prime}, d^{\prime}\right) \in M_{P}$, and suppose that $(a, b),\left(a^{\prime}, b^{\prime}\right)$ allow the reduction of $k_{P}(c, d), k_{P}\left(c^{\prime}, d^{\prime}\right)$ respectively. Then $(a, b),\left(a^{\prime}, b^{\prime}\right)$ are said to be compatible if $(a, b)-\left(a^{\prime}, b^{\prime}\right) \neq \mathbf{x}-\mathbf{y}$, for all $\mathbf{x}, \mathbf{y} \in F_{S}^{+} \backslash\{(c-$ $\left.a, d-b),\left(c^{\prime}-a^{\prime}, d^{\prime}-b^{\prime}\right)\right\}$ and all $\mathbf{x}, \mathbf{y} \in F_{S}^{-} \backslash\{(c-a, d-$ b), $\left.\left(c^{\prime}-a^{\prime}, d^{\prime}-b^{\prime}\right)\right\}$.

In the above definition we ignore the points $(c-$ $a, d-b),\left(c^{\prime}-a^{\prime}, d^{\prime}-b^{\prime}\right)$, as they provide multiple points in $M_{P}$. Furthermore, we say that a set of choices $(a, b)$, which allows the reduction of points in $M_{P}$, is a set of compatible choices if its elements are pairwise compatible.

We also note that in Proposition 3 we worked with the extra condition that $H(x, y)$, and all the progressively added monomials, have the same sign, say positive. This is motivated by the applicative purpose of regarding the addition of a binary ghost into real image data as a result of the combined addition of two complex ghosts.

Differently, since $H(x, y)=H^{+}(x, y)+H^{-}(x, y)$, we could look for a solution $H^{\prime}(x, y)$ related to the polynomial $H^{+}(x, y) F_{S}(x, y)$, and simply take $K(x, y)=$ $H^{\prime}(x, y)-H^{-}(x, y)$ to get a solution to Problem 1 .

The following theorem gives the theoretical basis for an algorithm that returns, if allowed, a binary ghost as an output.

Theorem 2 Let $S=\left\{u_{1}, u_{2}, u_{3}, u_{1}+u_{2} \pm u_{3}\right\}$ be a set of four lattice directions. Consider a polynomial $P(x, y)=$ $H(x, y) F_{S}(x, y)$, where $H(x, y)$ consists of monomials with positive coefficients. Suppose that for each $(c, d) \in$ $M_{P}$ there are $k_{P}(c, d)-1$ choices of pairs $(a, b)$, satisfying conditions $(A),(B),(C)$ and all such pairs $(a, b)$ form a set of compatible choices. Let $K(x, y)$ be the polynomial formed by the monomials $x^{a} y^{b}$ corresponding to all such pairs. Then $G(x, y)=(H(x, y)+K(x, y)) F_{S}(x, y)$ is a reduction of $P(x, y)$.

Proof We first show that for each pair of choices $(a, b)$, $\left(a^{\prime}, b^{\prime}\right)$, which allow the reduction of $k_{P}(c, d), k_{P}\left(c^{\prime}, d^{\prime}\right)$ respectively, the polynomial $Q(x, y)=\left(H(x, y)+x^{a} y^{b}+\right.$ $\left.x^{a^{\prime}} y^{b^{\prime}}\right) F_{S}(x, y)$ has no multiple points distinct from those in $P$, i.e. we have $M_{Q} \subseteq M_{P}$. Since $(a, b),\left(a^{\prime}, b^{\prime}\right)$ satisfy the conditions $(\mathrm{A}),(\mathrm{B})$ and $(\mathrm{C})$, the sets of multiple points of the polynomials $\left(H(x, y)+x^{a} y^{b}\right) F_{S}(x, y)$ and $\left(H(x, y)+x^{a^{\prime}} y^{b^{\prime}}\right) F_{S}(x, y)$ are contained in $M_{P}$. Thus 
we have to show that the multiple points of the polynomial $\left(x^{a} y^{b}+x^{a^{\prime}} y^{b^{\prime}}\right) F_{S}(x, y)$ are contained in $M_{P}$. This follows from the assumption that $(a, b),\left(a^{\prime}, b^{\prime}\right)$ are compatible. Finally, if for each $(c, d) \in M_{P}$ there are $k_{P}(c, d)-1$ monomials $x^{a} y^{b}$ in $K(x, y)$, then the statement follows.

In general, solutions to Problem 1 are provided by selecting several different pairs $(a, b)$ (which constitute the polynomial $K(x, y))$ satisfying Proposition 2. Each allowed choice reduces the multiplicity of at least one multiple point. Therefore, if $\eta$ is the number of choices, which reflects in the number of monomials of $K(x, y)$, required to get a reduction, it results

$\eta \leq \sum_{(c, d) \in M_{P}}\left(k_{P}(c, d)-1\right)=\sum_{(c, d) \in M_{P}} k_{P}(c, d)-\left|M_{P}\right|$,

where $\left|M_{P}\right|$ denotes the cardinality of $M_{P}$.

We resume the previous results in the following algorithm.

\section{Algorithm 3}

1: Input: A set $S$ of four lattice directions, and a polynomial $H(x, y)$ with positive coefficients.

2: Compute the polynomial $P(x, y)=H(x, y) F_{S}(x, y)$.

3: Let $M_{P}$ be the set of multiple points: For each $(c, d) \in$ $M_{P}$ select the pairs $(a, b)$ satisfying conditions $(A)$, (B), (C). Let $N(c, d)$ be the number of such pairs.

4: If $N(c, d)<k_{P}(c, d)-1$, then return: $N O R E D U C$ TION EXISTS.

5: Consider a polynomial $K(x, y)$ formed by the monomials corresponding to the selected pairs $(a, b)$.

6: If for every allowed selection, pairs are non-compatible, then return: NO REDUCTION EXISTS.

7: ELSE Compute the polynomial $G(x, y)=(H(x, y)+$ $K(x, y)) F_{S}(x, y)$.

8: Output: Binary ghost corresponding to the S-bad configuration $\left(G^{+}, G^{-}\right)$.

We implemented the algorithm in $\mathrm{C}$ programming language. Steps from 1 to 5 run in polynomial time. Steps 6-7 require to check compatibility from every couple of selected pairs, so that these steps can be expensive in terms of computational complexity. Therefore we implemented this part by means of a recursive function realizing a backtrack strategy (as a deep-first-search) which terminates as soon as a solution is found. In addition a branch and bound technique limits the range of admissible solutions and speeds up the running time. A systematic testing of the algorithm on real data will be investigated and discussed in a future work. However to a better understanding of the algorithm we show many different case-studies.

\section{Case-studies and discussion}

We now report and discuss on the application of Algorithm 3 to some selected examples. We usually work with the starting set of directions $S=\left\{u_{1}=(1,0), u_{2}=\right.$ $\left.(1,2), u_{3}=(0,1), u_{4}=(2,1)\right\}$, where the case $u_{1}+u_{2}-$ $u_{3}=u_{4}$ occurs. However, in Example 4 we briefly comment on different possible input set of directions.

\subsection{Applications I-On the feasible solutions}

At first, we show a case where our procedure returns no reduction.

Example 2 Consider the polynomial $H(x, y)=1+x y+$ $x^{2} y^{2}+x^{4} y^{3}+x^{2} y^{3}+x+y$, so that we have the following polynomial $P(x, y)=H(x, y) F_{S}(x, y)$

$$
\begin{aligned}
& \left(1+x y+x^{2} y^{2}+x^{4} y^{3}+x^{2} y^{3}+x+y\right) \\
& (x-1)(y-1)\left(x^{2} y-1\right)\left(x y^{2}-1\right)= \\
& =x^{8} y^{7}-x^{8} y^{6}-x^{7} y^{7}+x^{7} y^{6}-x^{7} y^{5}+ \\
& +x^{7} y^{4}+x^{6} y^{7}-x^{6} y^{6}+x^{6} y^{5}-x^{6} y^{4}-x^{5} y^{7} \\
& +x^{5} y^{6}+x^{5} y^{4}-x^{5} y^{3}-x^{4} y^{6}+x^{4} y^{5}- \\
& -x^{4} y^{3}+x^{4} y+x^{3} y^{6}-x^{3} y^{5}-x^{3} y^{4}+x^{3} y^{3} \\
& -x^{2} y^{4}+x^{2} y^{3}+2 x^{2} y^{2}-x^{2} y-x^{2}+x y^{4}-x y^{2}-y^{2}+1
\end{aligned}
$$

The only multiple point $\mathbf{m}=(2,2)$ can be obtained from points in $F_{S}$ as follows

$$
\mathbf{m}=(0,0)+(2,2)
$$$$
\text { translation of }(2,2) \in F_{S}^{+} \text {(double point) }
$$

by means of the monomial 1

$\mathbf{m}=(1,1)+(1,1)$ translation of $(1,1) \in F_{S}^{+}$

by means of the monomial $x y$

$$
\begin{aligned}
& \mathbf{m}=(2,2)+(0,0) \text { translation of }(0,0) \in F_{S}^{+} \\
& \text {by means of the monomial } x^{2} y^{2}
\end{aligned}
$$$$
\mathbf{m}=(0,1)+(2,1) \text { translation of }(2,1) \in F_{S}^{-}
$$$$
\text { by means of the monomial } y
$$$$
\mathbf{m}=(1,0)+(1,2) \text { translation of }(1,2) \in F_{S}^{-}
$$

by means of the monomial $x$.

Note that no reduction is allowed. In fact, we can easily see that, for each choice of $\mathbf{n} \in F_{S}^{-}$, the point $(a, b)=$ $(2,2)-\mathbf{n}$ does not satisfy conditions (9) or (10) (or both), so that its multiplicity cannot be reduced without adding new multiplicities as asked in Problem 1. 
Now we give an example in which Algorithm 3 returns a solution.

Example 3 Consider the polynomial $H(x, y)=1+x y+$ $x^{2} y^{2}$. We have

$$
\begin{aligned}
& P(x, y)=H(x, y) F_{S}(x, y)= \\
& =\left(1+x y+x^{2} y^{2}\right)(x-1)(y-1)\left(x^{2} y-1\right)\left(x y^{2}-1\right)= \\
& =x^{6} y^{6}-x^{6} y^{5}-x^{5} y^{6}+2 x^{5} y^{5}-2 x^{5} y^{4}+ \\
& +x^{5} y^{3}-2 x^{4} y^{5}+4 x^{4} y^{4}-3 x^{4} y^{3}+x^{4} y^{2}+x^{3} y^{5} \\
& -3 x^{3} y^{4}+4 x^{3} y^{3}-3 x^{3} y^{2}+x^{3} y+x^{2} y^{4}- \\
& -3 x^{2} y^{3}+4 x^{2} y^{2}-2 x^{2} y+x y^{3}- \\
& -2 x y^{2}+2 x y-x-y+1
\end{aligned}
$$

The set of coefficients different from $\{-1,0,1\}$ is $\{-3,-2,2,4\}$, which represents the levels of corruption of the binary ghost. The related digital image is shown in Figure 3(a).

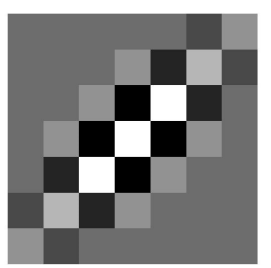

(a)

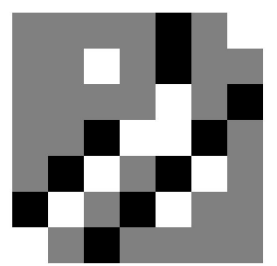

(b)
Fig. 3: (a) A corrupted ghost. There are 4 different levels of corruption, corresponding to the set of coefficients different from $\{-1,0,1\}$ in the associated polynomial representation. (b) A corresponding binary ghost.

A complete reduction of $P(x, y)$ is obtained just by three choices, corresponding to the selected pairs $(1,2)$, $(2,1)$, and $(1,0)$. This provides the following reduction

$$
\begin{aligned}
& G(x, y)=\left(H(x, y)+x y^{2}+x^{2} y+x\right) F_{S}(x, y)= \\
& =\left(1+x y+x^{2} y^{2}+x y^{2}+x^{2} y+x\right) \\
& (x-1)(y-1)\left(x^{2} y-1\right)\left(x y^{2}-1\right)= \\
& =x^{6} y^{6}-x^{6} y^{4}-x^{5} y^{3}+x^{5} y^{2}- \\
& -x^{4} y^{6}-x^{4} y^{5}+x^{4} y^{4}+x^{4} y^{3}-x^{4} y^{2} \\
& +x^{4} y+x^{3} y^{3}-x^{3} y+x^{2} y^{5}-x^{2} y^{3}+ \\
& +x^{2} y^{2}-x^{2}-x y^{2}+x y-y+1
\end{aligned}
$$

Figure 3(b) shows the corresponding binary ghost image. Let us explain the result. Note that
- the pair $(1,2)$ can be selected as in Proposition 3 for the multiple positive points $\mathbf{m}_{2}=(2,2), \mathbf{m}_{3}=$ $(3,3), \mathbf{m}_{4}=(4,4), \mathbf{m}_{5}=(5,5)$. The same pair $(1,2)$ can be selected also for the multiple negative points $\mathbf{m}_{6}=(1,2), \mathbf{m}_{8}=(2,3), \mathbf{m}_{10}=(3,4), \mathbf{m}_{11}=$ $(4,3), \mathbf{m}_{12}=(4,5)$,

- the pair $(2,1)$ can be selected for the multiple positive points $\mathbf{m}_{2}=(2,2), \mathbf{m}_{3}=(3,3), \mathbf{m}_{4}=(4,4)$, $\mathbf{m}_{5}=(5,5)$. The same pair $(2,1)$ can be selected also for the multiple negative points $\mathbf{m}_{7}=(2,1)$, $\mathbf{m}_{9}=(3,2), \mathbf{m}_{10}=(3,4), \mathbf{m}_{11}=(4,3), \mathbf{m}_{13}=$ $(5,4)$,

- the pair $(1,0)$ can be selected for the multiple positive points $\mathbf{m}_{1}=(1,1), \mathbf{m}_{2}=(2,2), \mathbf{m}_{3}=(3,3)$, $\mathbf{m}_{4}=(4,4)$. The same pair $(1,0)$ can be selected also for the multiple negative points $\mathbf{m}_{7}=(2,1)$, $\mathbf{m}_{8}=(2,3), \mathbf{m}_{9}=(3,2), \mathbf{m}_{10}=(3,4), \mathbf{m}_{11}=$ $(4,3), \mathbf{m}_{13}=(5,4)$.

Therefore, the points $(2,2),(3,3)$, and $(4,4)$, having multiplicity 4 , are covered 3 times, the points $(4,3)$, $(3,4),(3,2)$, and $(2,3)$, having multiplicity 3 , are covered 3 or 2 times, and all the double points are covered 1 or 2 times. Consequently $\left(H(x, y)+x y^{2}+x^{2} y+\right.$ $x) F_{S}(x, y)$ represents the corresponding reduced polynomial.

According to Proposition 4, the number of choices required to get a reduction can be considerably less than the upper bound provided by (12), since condition (11) often (but not always, see Example 5) holds. Moreover, different reductions could be determined by Algorithm 3 for a same starting polynomial, associated to different orders in the selections of the allowed pairs. For instance, in Example 3, a further possible reduction is given by

$$
\begin{aligned}
& G^{\prime}(x, y)= \\
& =\left(1+x y+x^{2} y^{2}+x^{2} y+x+y\right) \\
& (x-1)(y-1)\left(x^{2} y-1\right)\left(x y^{2}-1\right)= \\
& =x^{6} y^{6}-x^{6} y^{4}-x^{5} y^{6}+x^{5} y^{5}-x^{5} y^{3}+ \\
& +x^{5} y^{2}-x^{4} y^{5}+x^{4} y^{4}-x^{4} y^{2} \\
& +x^{4} y-x^{3} y^{4}+x^{3} y^{3}+x^{3} y^{2}-x^{3} y+ \\
& +x^{2} y^{2}-x^{2}+x y^{4}-x y^{2}-y^{2}+1
\end{aligned}
$$

Example 4 Different examples can be easily provided by changing the input set $S=\left\{u_{1}, u_{2}, u_{3}, u_{4}\right\}$ and the polynomial $H(x, y)$. In particular, if $S$ is selected such that $u_{4} \neq u_{1}+u_{2} \pm u_{3}$ then condition (C) is not necessary, since $F_{S}$ does not have multiple points. Note that $u_{4} \neq u_{1}+u_{2} \pm u_{3}$ must be checked for any permutations of the indices. For instance, if $S=\left\{u_{1}=(1,1), u_{2}=\right.$ 
$\left.(1,2), u_{3}=(1,3), u_{4}=(1,4)\right\}$, it is $u_{1}+u_{2}+u_{3}=(3,6)$, and $u_{1}+u_{2}-u_{3}=(1,0)$, so that $u_{4} \neq u_{1}+u_{2} \pm u_{3}$. However, by exchanging indices 1 and 3 , we have $u_{3}+u_{2}-$ $u_{1}=(1,4)=u_{4}$, and consequently $F_{S}$ has still a double point. Differently, consider $S=\left\{u_{1}=(1,1), u_{2}=\right.$ $\left.(1,2), u_{3}=(1,3), u_{4}=(1,5)\right\}$. In this case no permutation of indices allows $u_{4}=u_{1}+u_{2} \pm u_{3}$, so that $F_{S}$ has no multiple points. Assume such set $S$, and the polynomial $H(x, y)=x y+x y^{2}$ in the input of Algorithm 3, so that

$$
\begin{aligned}
& P(x, y)=H(x, y) F_{S}(x, y)= \\
& =\left(x y+x y^{2}\right)(x y-1)\left(x y^{2}-1\right)\left(x y^{3}-1\right)\left(x y^{5}-1\right)= \\
& =x^{5} y^{13}+x^{5} y^{12}-x^{4} y^{12}-2 x^{4} y^{11}-2 x^{4} y^{10}-x^{4} y^{9} \\
& -x^{4} y^{8}-x^{4} y^{7}+x^{3} y^{1} 0+2 x^{3} y^{9}+2 x^{3} y^{8}+2 x^{3} y^{7} \\
& +2 x^{3} y^{6}+2 x^{3} y^{5}+x^{3} y^{4}-x^{2} y^{7}-x^{2} y^{6}-x^{2} y^{5} \\
& -2 x^{2} y^{4}-2 x^{2} y^{3}-x^{2} y^{2}+x y^{2}+x y .
\end{aligned}
$$

A complete reduction can be obtained, for instance, just adding $K(x, y)=1+x^{2} Y^{4}$ to $H(x, y)$. In fact, according to Proposition 3 , the pair $(0,0)$ can be selected for the multiple points $(2,3),(2,4),(3,5),(3,6),(3,8)$, $(3,9),(4,11)$, and the pair $(2,4)$ can be selected for the multiple points $(3,5),(3,7)$, and $(4,10)$. The resulting binary ghost is the following

$$
\begin{aligned}
& G(x, y)=\left(H(x, y)+1+x^{2} y^{4}\right) F_{S}(x, y)= \\
& =\left(x y^{2}+x y+1+x^{2} y^{4}\right) \\
& (x y-1)\left(x y^{2}-1\right)\left(x y^{3}-1\right)\left(x y^{5}-1\right)= \\
& =x^{6} y^{15}-x^{5} y^{14}-x^{5} y^{10}-x^{4} y^{10}+x^{3} y^{8}+x^{3} y^{7} \\
& +x^{3} y^{5}+x^{3} y^{4}+x^{2} y^{8}-x^{2} y^{3}-x^{2} y^{2}-x y^{5}-x y^{3}+1 .
\end{aligned}
$$

As a further remark, we point out that, in view of Theorem 1, which is valid for any choice $S$, the same Algorithm 3 can be applied to set $S$ of higher cardinality, up to checking conditions (C) for any possible multiple point of $F_{S}$.

\subsection{Applications II-On the size of the solutions}

Now, we determine upper bounds for the degrees of any solution to Problem 1.

Theorem 4 Assume $G(x, y)$ is a reduction of $P(x, y)=$ $H(x, y) F_{S}(x, y)$. Then

$$
\operatorname{deg}_{x} G(x, y) \leq \operatorname{deg}_{x} P(x, y)+\operatorname{deg}_{x} F_{S}(x, y)
$$

and

$$
\operatorname{deg}_{y} G(x, y) \leq \operatorname{deg}_{y} P(x, y)+\operatorname{deg}_{y} F(x, y) .
$$

Proof Since $G(x, y)$ is a reduction of $P(x, y)$, then $G(x, y)=$ $(H(x, y)+K(x, y)) F_{S}(x, y)$, where $K(x, y)$ consists of monomials $x^{a} y^{b}$ with $(a, b)=(c, d)-\mathbf{r},(c, d) \in M_{P}$, and $\mathbf{r} \in F_{S}$ (see condition $\left.(\mathrm{A})\right)$. Since $(c, d) \in P$, there exists $(\alpha, \beta) \in H$, such that $(c, d)=\mathbf{q}+(\alpha, \beta)$, where $\mathbf{q} \in F_{S}$. Therefore $(a, b)=(\alpha, \beta)+\mathbf{q}-\mathbf{r}=(\alpha, \beta)+\mathbf{d}$, where $\mathbf{d} \in F_{S}-F_{S}$. This implies

$$
\begin{aligned}
& a \leq \alpha+\operatorname{deg}_{x} F_{S}(x, y) \\
& b \leq \beta+\operatorname{deg}_{y} F_{S}(x, y),
\end{aligned}
$$

for all $(a, b)$ such that $x^{a} y^{b}$ is a monomial of $K(x, y)$. Therefore, we get

$\operatorname{deg}_{x} K(x, y) \leq \operatorname{deg}_{x} H(x, y)+\operatorname{deg}_{x} F_{S}(x, y)=\operatorname{deg}_{x} P(x, y)$ $\operatorname{deg}_{y} K(x, y) \leq \operatorname{deg}_{y} H(x, y)+\operatorname{deg}_{y} F_{S}(x, y)=\operatorname{deg}_{y} P(x, y)$.

From this we get

$\operatorname{deg}_{x} K(x, y)+\operatorname{deg}_{x} F_{S}(x, y) \leq \operatorname{deg}_{x} P(x, y)+\operatorname{deg}_{x} F_{S}(x, y)$ $\operatorname{deg}_{y} K(x, y)+\operatorname{deg}_{y} F_{S}(x, y) \leq \operatorname{deg}_{y} P(x, y)+\operatorname{deg}_{y} F_{S}(x, y)$.

Since $G(x, y)=(H(x, y)+K(x, y)) F_{S}(x, y)=P(x, y)+$ $K(x, y) F_{S}(x, y)$, it results

$\operatorname{deg}_{x} G(x, y)=\max \left\{\operatorname{deg}_{x} P(x, y), \operatorname{deg}_{x} K(x, y) F_{S}(x, y)\right\}=$ $=\max \left\{\operatorname{deg}_{x} P(x, y), \operatorname{deg}_{x} K(x, y)+\operatorname{deg}_{x} F_{S}(x, y)\right\}$

$\operatorname{deg}_{y} G(x, y)=\max \left\{\operatorname{deg}_{y} P(x, y), \operatorname{deg}_{y} K(x, y) F_{S}(x, y)\right\}=$ $=\max \left\{\operatorname{deg}_{y} P(x, y), \operatorname{deg}_{y} K(x, y)+\operatorname{deg}_{y} F_{S}(x, y)\right\}$,

and (13), (14) follow.

Remark 2 If all the monomials in $H(x, y)$ have coefficient 1 , then also the following lower bounds hold

$$
\operatorname{deg}_{x} P(x, y) \leq \operatorname{deg}_{x} G(x, y)
$$

and

$$
\operatorname{deg}_{y} P(x, y) \leq \operatorname{deg}_{y} G(x, y) .
$$

If equality holds in (15) and (16), then the minimal grid containing $P$ also contains the $S$-bad configuration $\left(G^{+}, G^{-}\right)$(see for instance Example 3). This means that the maximal size of the sets which are uniquely determined by the X-rays in the directions in $S$ must be smaller than the size of $P$.

In the reduction provided in the previous examples the lower bounds in (15) and (16) hold. However, this is not always the case, as it is shown by the following example, where we shall assume $S=\left\{u_{1}=(1,0), u_{2}=\right.$ $\left.(1,2), u_{3}=(0,1), u_{4}=(2,1)\right\}$, where the case $u_{1}+u_{2}-$ $u_{3}=u_{4}$ occurs. 
Example 5 Assume, for instance the polynomial $P(x, y)=$ of the form $H(x, y) F_{S}(x, y)$ computing a polynomial $\left(1+x^{5}\right) F_{S}(x, y)$, so that

$$
\begin{aligned}
& P(x, y)=\left(1+x^{5}\right)(x-1)(y-1)\left(x^{2} y-1\right)\left(x y^{2}-1\right)= \\
& x^{9} y^{4}-x^{9} y^{3}-x^{8} y^{4}+x^{8} y^{3}-x^{8} y^{2}+x^{8} y-x^{7} y^{3}+ \\
& +2 x^{7} y^{2}-x^{7} y+x^{6} y^{3}-x^{6} y^{2}+x^{6} y-x^{6}-x^{5} y+ \\
& +x^{5}+x^{4} y^{4}-x^{4} y^{3}-x^{3} y^{4}+x^{3} y^{3}-x^{3} y^{2}+x^{3} y- \\
& -x^{2} y^{3}+2 x^{2} y^{2}-x^{2} y+x y^{3}-x y^{2}+x y-x-y+1 .
\end{aligned}
$$

We have two double points, both positive. A first choice is $(a, b)=(7,2)-(3,2)=(4,0)$, which removes the double point $(c, d)=(7,2)$, and it gives

$$
\begin{aligned}
& \left(1+x^{5}+x^{4}\right)(x-1)(y-1)\left(x^{2} y-1\right)\left(x y^{2}-1\right)= \\
& x^{9} y^{4}-x^{9} y^{3}-x^{8} y^{2}+x^{8} y-x^{7} y^{4}+x^{7} y^{2}+x^{6} y^{2}-x^{6}+ \\
& +x^{5} y^{3}-x^{5} y^{2}+x^{4} y^{4}-x^{4} y^{3}-x^{4} y+x^{4}-x^{3} y^{4}+x^{3} y^{3}- \\
& -x^{3} y^{2}+x^{3} y-x^{2} y^{3}+2 x^{2} y^{2}-x^{2} y+x y^{3}- \\
& -x y^{2}+x y-x-y+1
\end{aligned}
$$

The multiplicity of the remaining double point $(c, d)=$ $(2,2)$ can be reduced by selecting $(a, b)=(2,2)-(2,1)=$ $(0,1)$. This provides the following reduction

$$
\begin{aligned}
& G(x, y)= \\
& \left(1+x^{5}+x^{4}+y\right)(x-1) \\
& (y-1)\left(x^{2} y-1\right)\left(x y^{2}-1\right)= \\
& x^{9} y^{4}-x^{9} y^{3}-x^{8} y^{2}+x^{8} y-x^{7} y^{4}+x^{7} y^{2}+ \\
& +x^{6} y^{2}-x^{6}+x^{5} y^{3}-x^{5} y^{2}+x^{4} y^{5}-x^{4} y^{3}- \\
& -x^{4} y+x^{4}-x^{3} y^{5}+x^{3} y-x^{2} y^{4}+x^{2} y^{3}+ \\
& +x^{2} y^{2}-x^{2} y+x y^{4}-x-y^{2}+1
\end{aligned}
$$

Note that $\operatorname{deg}_{y} G(x, y)=5>\operatorname{deg}_{y} P(x, y)$, and the lower bound in (16) does not hold. This example also shows that, being $\eta=2$, in general, the upper bound (12) on the required number of choices needed to get a reduction cannot be lowered.

\section{Conclusions}

In this paper, a new algebraic approach to the construction of minimal bad configurations has been introduced. We determined a general theoretical upper bound for the size of the smallest bad configurations associated to a given set $S$ of lattice directions. We proved that in the special but interesting case of four directions, the upper bound can be considerably improved. Moreover, we provided an algorithm for removing the multiplicities of a given input polynomial $(K(x, y)+H(x, y)) F_{S}(x, y)$ with coefficients in $\{-1,0,1\}$. As we showed that finding bad configurations (i.e. binary ghosts) is equivalent to finding multiples of a suitable polynomial in two variables having only coefficients from the set $\{-1,0,1\}$, our procedure furnishes an explicit method for the construction of ghosts from projection data, and consequently could be of interest in image processing. Indeed, adding binary ghosts causes the weakest perturbation of the original image (see for instance Figure 4) and provides a way to demonstrate, for example, if any algorithm produces medical-relevant reconstructions as shown in [13].
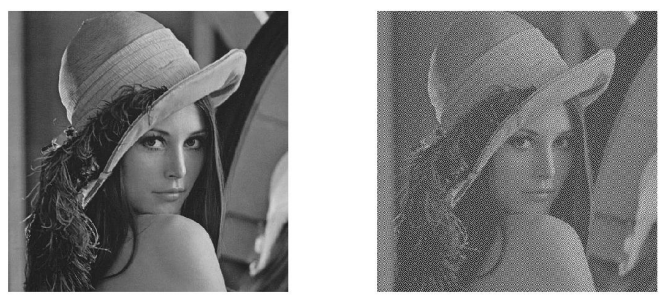

Fig. 4: Left: original Image. Right: corruption of the original image by addition of a binary ghost.

We illustrated our method by means of several examples, and for different input polynomials we showed the corresponding output. Since the coupling between image structure and its projected versions is the key to exploit redundancy, we feel that our new approach might be of interest also in view of efficient algorithms to encode data.

\section{Acknowledgements}

Research of the third author was supported in part by the OTKA grants K100339, NK101680. The publication was supported by the TÁMOP-4.2.2.C-11/1/KONV2012-0001 project. The project has been supported by the European Union, co-financed by the European Social Fund.

\section{References}

1. P. Borwein AND T. ERdÉlyi, On the zeros of polynomials with restricted coefficients, Illinois J. Math. 41 (1997), no. 4, pp. 667-675 (URL: http://projecteuclid.org/euclid.ijm/1256068987).

2. R. J. Gardner, Geometric Tomography. Cambridge University Press, New York (1995). Second edition, 2006.

3. G. T. Herman and A. Kuba, Advances in Discrete Tomography and Its Applications, Appl. Numer. Harmon. Anal., Birkhäuser Boston, Boston, MA, 2007. 
4. K. J. Batenburg, W. J. Palenstijn, P. Balázs and J. SiJBERS, Dynamic angle selection in binary tomography, Computer Vision and Image Understanding, 117 (4), (2013), 306-318 (http://dx.doi.org/10.1016/j.cviu.2012.07.005)

5. D. J. Witheya, W. PedryczB, And Z. J. Kolesb, Dynamic edge tracing: Boundary identification in medical images, Computer Vision and Image Understanding, 113 (10), (2009), 10391052 (http://dx.doi.org/10.1016/j.cviu.2009.07.003)

6. J. Gregor AND T. BENSON, Computational analysis and improvement of SIRT, IEEE Trans. Med. Imaging, 27(7), (2008), 918-924 (http://dx.doi.org/ 10.1109/TMI.2008.923696)

7. I. Debled-Rennesson, E. Domenjoud, B. KerAutret, P. Even, Special Issue on Discrete Geometry for Computer Imagery, Computer Vision and Image Understanding, 117 (4), (2013), 305 (http://dx.doi.org/10.1016/j.cviu.2013.01.009).

8. S. van Aert, K.J. Batenburg, M.D. Rossell, R. Erni, AND G. VAN TENDElOO, Three-dimensional atomic imaging of crystalline nanoparticles, Nature, 470, (2011), 374-377 (http://www.nature.com/nature/journal/v470/n7334/ abs/nature09741.html).

9. A. H. Andersen And A. C. KAK., Simultaneous Algebraic Reconstruction Tecnique (SART): a superior implementation of the ART algorithm, Ultrason. Img., 6 (1984) 81-94 (doi: 10.1016/0161-7346(84)90008-7

10. A. C. KAK AND M. Slaney, Principles of Computerized Tomographic Imaging, SIAM, 2001 ( http://dx.doi.org/10.1137/1.9780898719277)

11. R. Gordon R AND G. T. HeRman Reconstruction of pictures from their projections, Commun ACM. 14 (1971), 759768 (doi:10.1145/362919.362925).

12. G. T. HERMAN, Image Reconstruction from Projections: The Fundamentals of Computerized Tomography, New York: Academic Press; 1980.

13. G. T. HERman AND R DAVIdI, Image Reconstruction from a Small Number of Projections, Inverse Problems 24 (4) 2008 (doi:10.1088/0266-5611/24/4/045011).

14. K. J. Batenburg And J. SiJbers, DART: A Practical Reconstruction Algorithm for Discrete Tomography, IEEE Transactions on Image Processing, 20 (9) (september 2011), 2542-53 ( http://dx.doi.org/10.1109/TIP.2011.2131661)

15. L. VArga, L. G. Nyúl, A. NAgy and P. Balázs, Local Uncertainty in Binary Tomographic Reconstruction, Proceedings of the IASTED International Conference on Signal Processing, Pattern Recognition and Applications, (2013), Innsbruck, Austria, 490-496 ( http://dx.doi.org/10.2316/P.2013.798-067)

16. L. VARGA, P. BALÁzs, A. NAGY, Directiondependency of binary tomographic reconstruction algorithms, Graph. Models, (73), (2011), 365-375 (http://dx.doi.org/10.1016/j.gmod.2011.06.006)

17. L. VArga, P. BALÁzs, A. NAGY, Projection selection dependency in binary tomography, Acta Cybernet., 20(1), (2011), 167-187 (http://dl.acm.org/citation.cfm?id=2336107.2336118)

18. Z. Zheng AND K. Mueller, Identifying Sets of Favorable Projections for Few-View Low-Dose Cone-Beam CT Scanning, in: 11th International Meeting on Fully ThreeDimensional Image Reconstruction in Radiology and $\mathrm{Nu}$ clear Medicine, 2011, 314-317

19. M. B. KATZ, Questions of uniqueness and resolution in reconstruction from projections, Lecture Notes in Biomathematics, 26, Springer-Verlag, Berlin-New York, (1978).
20. P. C. Fishburn, J. C. Lagarias, J. A. Reeds, And L. A. SHEPP, Sets uniquely determined by projections on axes II. Discrete case, Discrete Math. 91 (1991), pp.149159 (doi:10.1016/0012-365X(91)90106-C).

21. H. J. Ryser, Combinatorial properties of matrices of zeros and ones, Canad. J. Math. 9(1957), 371-377 (doi:/10.4153/CJM-1957-044-3).

22. S. K. CHANG, The reconstruction of binary patterns from their projections, Commun. ACM 14 (1971), 21-24 (doi:10.1145/362452.362471).

23. G. G. Lorentz, A problem of plane measure, Amer. J. Math. 71 (1949), 417-426 (URL:http://www.jstor.org/stable/2372255).

24. R. J. GARDNER AND P. GRITZMAnN, Uniqueness and complexity in discrete tomography, in: Discrete Tomography: Foundations, Algorithms and Application, ed. by G. T. Herman and A. Kuba, Birkhäuser, Boston, 1999, pp. 85-113.

25. L. Hajdu and R. Tijdeman, Algebraic aspects of discrete tomography, J. Reine angew. Math $\mathbf{5 3 4}$ (2001), 119-128 (doi:10.1515/crll.2001.037).

26. P. Dulio AND C. PERI, Discrete tomography for inscribable lattice sets, Discrete Applied Mathematics, 161 (2013), 1959-1974 (doi:10.1016/j.dam.2013.03.025).

27. S. Brunetti, P. Dulio, And C. Peri, On the NonAdditive Sets of Uniqueness in a Finite Grid, DGCI 2013, LNCS 7749, (2013) 288-299 (doi:10.1007/978-3642-37067-0_25).

28. S. Brunetti, P. Dulio, And C. Peri, Characterization of $\{-1,0,1\}$ valued functions in discrete tomography under sets of four directions, DGCI 2011 LNCS 6607, (2011), 394-405 (doi:10.1007/978-3-642-19867-0_33).

29. S. Brunetti, P. Dulio, and C. Peri, Discrete Tomography determination of bounded lattice sets from four Xrays, Discrete Applied Mathematics 161 (15) (2013), 22812292 (doi:10.1016/j.dam.2012.09.010) .

30. L. HAJDU, Unique reconstruction of bounded sets in discrete tomography, Electron. Notes Discrete Math., 20 (2005) 15-25 (doi:10.1016/j.endm.2005.04.002).

31. R. J. GARDNer And P. GRITZMAnn, Discrete tomography: Determination of finite sets by X-rays, Trans. Amer. Math. Soc. 349 (1997), pp. 2271-2295 (doi:10.1090/S0002-994797-01741-8).

32. R. J. Gardner and P. McMullen, On Hammer's X-ray Problem, J. London Math. Soc. 21 (2) (1980) 171-175 (doi: 10.1112/jlms/s2-21.1.171).

33. S. BRUnetTi AND A. DAURAT, An algorithm reconstructing convex lattice sets, Theoret. Comp. Sci. 304 (2003), pp. 3557 (doi:10.1016/S0304-3975(03)00050-1.

34. P. Dulio, Convex decomposition of $U$-polygons, Theoretical Computer Science, 406/1-2, (2008), 80-89 (doi:10.1016/j.tcs. 2008.06.008).

35. P. Dulio AND C. PERI, On the geometric structure of lattice U-polygons, Discrete Math., 307/19-20 (2007), 2330-2340 (doi: 10.1016/j.disc.2006.09.044).

36. C. HUCK AND M. SPIEß, Solution of a uniqueness problem in the discrete tomography of algebraic Delone sets, J. Reine Angew. Math. 677 (2013), 199-224 (doi:10.1515/crelle.2012.026).

37. J. Matoušek, A. Přílvětivý, And P. S̆Kovroñ, How many points can be reconstructed from $k$ projections?, SIAM J. Discrete Math. 22 (4), (2008), 1605-1623 (doi:10.1137/080715706).

38. E. Barcucci, A. Del Lungo, M. Nivat, and R. Pinzani, $X$-rays characterizing some classes of discrete sets Linear Algebra Appl., 339 (2001), 3-21 (doi:10.1016/S00243795(01)00431-1). 
39. G. Bianchi And M. Longinetti, Reconstructing plane sets from projections, Discrete Comput. Geom., 5 (1990), $223-$ 242 (doi:10.1007/BF02187787).

40. R. J. Gardner, X-rays of polygons, Discrete Comput. Geom., 7 (1992) 281-293 (doi:10.1007/BF02187842).

41. I. Svalbe And S. Chandra Growth of Discrete Projection Ghosts Created by Iteration, DGCI 2011, LNCS 6607, (2011), 406416 (doi:10.1007/978-3-642-19867-0_34).

42. I. Svalbe and N. Normand, Properties of minimal ghosts, DGCI 2011, LNCS 6607, (2011), 417-428 (doi:10.1007/978-3-642-19867-0_35).

43. I. Svalbe, N. Nazareth, and S. Chandra, On constructing minimal ghosts, in: DICTA 2010, Sydney, Australia (December 2010), (2010), 276-281 (doi:10.1109/DICTA.2010.56).

44. S. Chandra, I. Svalbe and J. P. Guédon, An Exact, Non-iterative Mojette Inversion Technique Utilising Ghosts, LNCS, 4992, Springer Berlin/ Heidelberg, (2008), 401412 